\title{
Stimulated Raman adiabatic passage in a multilevel atom
}

\author{
A. D. Boozer \\ Norman Bridge Laboratory of Physics 12-33, California Institute of Technology, Pasadena, California 91125, USA
}

(Received 7 November 2007; published 11 February 2008)

\begin{abstract}
We present a general formalism for describing stimulated Raman adiabatic passage in a multilevel atom. The atom is assumed to have two ground state manifolds $a$ and $b$ and an excited state manifold $e$, and the adiabatic passage is carried out by resonantly driving the $a-e$ and $b-e$ transitions with time-dependent fields. Our formalism gives a complete description of the adiabatic passage process and can be applied to systems with arbitrary numbers of degenerate states in each manifold and arbitrary couplings of the $a-e$ and $b-e$ transitions. We illustrate the formalism by applying it to both a simple toy model and to adiabatic passage in the cesium atom.
\end{abstract}

DOI: 10.1103/PhysRevA.77.023411

PACS number(s): 37.10.Jk

\section{INTRODUCTION}

A number of techniques for coherently manipulating atomic systems are based on the idea of adiabatically varying the parameters of a time-dependent Hamiltonian. One such example is the technique of stimulated Raman adiabatic passage (STIRAP), which can be used to coherently transfer population between the two ground states of a three-level atom in the lambda configuration [1-3]. The STIRAP technique involves driving the atom with a pair of timedependent fields that couple the two ground states to the excited state and relies on the fact that the Hamiltonian for the system has an instantaneous eigenstate, called the dark state, that contains no excited state component. In general this dark state is a superposition of the two ground states, where the overlap of the dark state with each ground state depends on the ratio of the powers in the two fields. Population is transfered from the first ground state to the second by adiabatically varying these powers in such a way that initially the dark state overlaps entirely with the first ground state, during the process the dark state is a superposition of the two ground states, and after the process has been completed the dark state overlaps entirely with the second ground state. The STIRAP technique has been studied experimentally [4-10] and has formed the basis for numerous theoretical proposals [11-14]. In addition, STIRAP has been generalized to multilevel systems [15-18], opening up new possibilities for creating superposition states [19-23].

Here we present a general formalism for describing STIRAP in a multilevel atom. The atom is assumed to have two ground state manifolds $a$ and $b$ and an excited state manifold $e$, where each manifold consists of an arbitrary number of degenerate states, and the adiabatic passage is carried out by resonantly driving the $a-e$ and $b-e$ transitions with timedependent fields. The Hamiltonian for the system is

$$
H=\frac{\Omega}{2} \cos \theta\left(A_{b}+A_{b}^{\dagger}\right)-\frac{\Omega}{2} \sin \theta\left(A_{a}+A_{a}^{\dagger}\right),
$$

where $\Omega$ is a time-independent Rabi frequency that characterizes the strength of the fields, $\theta$ is a time-dependent parameter that is varied to carry out the adiabatic passage, and $A_{a}$ and $A_{b}$ are lowering operators that connect states in manifold $e$ to states in manifolds $a$ and $b$. We sweep $\theta$ from 0 to $\pi / 2$ to transfer states from manifold $a$ to manifold $b$, and we sweep $\theta$ from $\pi / 2$ to 0 to transfer states from manifold $b$ to manifold $a$.

Let us denote the Hilbert spaces for manifolds $a, b$, and $e$ by $\mathcal{H}_{a}, \mathcal{H}_{b}$, and $\mathcal{H}_{e}$. We will show that $\mathcal{H}_{a}$ and $\mathcal{H}_{b}$ can be decomposed as follows:

$$
\begin{aligned}
& \mathcal{H}_{a}=\mathcal{H}_{a}^{\lambda} \oplus \mathcal{H}_{a}^{d} \oplus \mathcal{H}_{a}^{\prime}, \\
& \mathcal{H}_{b}=\mathcal{H}_{b}^{\lambda} \oplus \mathcal{H}_{b}^{d} \oplus \mathcal{H}_{b}^{\prime} .
\end{aligned}
$$

Under adiabatic passage from $a$ to $b$, states in $\mathcal{H}_{a}^{\lambda}$ coherently evolve into states in $\mathcal{H}_{b}^{\lambda}$, states in $\mathcal{H}_{a}^{d}$ remain unchanged, and states in $\mathcal{H}_{a}^{\prime}$ are driven to the excited state manifold and scatter incoherently. Similarly, for adiabatic passage from $b$ to $a$, states in $\mathcal{H}_{b}^{\lambda}$ coherently evolve into states in $\mathcal{H}_{a}^{\lambda}$, states in $\mathcal{H}_{b}^{d}$ remain unchanged, and states in $\mathcal{H}_{b}^{\prime}$ are driven to the excited state manifold and scatter incoherently. The coherent evolution from $\mathcal{H}_{a}^{\lambda}$ to $\mathcal{H}_{b}^{\lambda}$ is described by a unitary transformation $U: \mathcal{H}_{a}^{\lambda} \rightarrow \mathcal{H}_{b}^{\lambda}$, and the coherent evolution from $\mathcal{H}_{b}^{\lambda}$ to $\mathcal{H}_{a}^{\lambda}$ is described by the unitary transformation $U^{\dagger}: \mathcal{H}_{b}^{\lambda} \rightarrow \mathcal{H}_{a}^{\lambda}$. Given arbitrary operators $A_{a}$ and $A_{b}$, our goal is to perform the Hilbert space decompositions described in Eqs. (2) and (3) and to calculate the unitary transformation $U$.

The paper is organized as follows. In Sec. II, we show how to preform the Hilbert space decompositions described in Eqs. (2) and (3). In Sec. III, we calculate the unitary transformation $U: \mathcal{H}_{a}^{\lambda} \rightarrow \mathcal{H}_{b}^{\lambda}$, and consider in detail the special case $\operatorname{dim} \mathcal{H}_{a}^{\lambda}=\operatorname{dim} \mathcal{H}_{b}^{\lambda}=2$. The formalism developed in Secs. II and III gives a complete description of the adiabatic transfer process, and in Sec. IV we illustrate this formalism by applying it to a simple toy model. Finally, in Sec. V we use the formalism to analyze adiabatic passage in the cesium atom.

\section{HILBERT SPACE DECOMPOSITIONS}

We will first show how to perform the Hilbert space decompositions described in Eqs. (2) and (3) for a given pair of atomic lowering operators $A_{a}$ and $A_{b}$. Let $\mathcal{H}_{a}^{d}$ be the space of states in manifold $a$ that are dark to the $a \rightarrow e$ transition, let $\mathcal{H}_{b}^{d}$ be the space of states in manifold $b$ that are dark to the $b \rightarrow e$ transition, and let $\mathcal{H}_{e}^{d}$ be the space of states in manifold 
$e$ that are dark to the $e \rightarrow b$ transition. Note that $\mathcal{H}_{a}^{d}, \mathcal{H}_{b}^{d}$, and $\mathcal{H}_{e}^{d}$ are just the null spaces of the operators $A_{a}^{\dagger}, A_{b}^{\dagger}$, and $A_{b}$ :

$$
\begin{aligned}
& \left.\mathcal{H}_{a}^{d}=\left\{\left|\psi_{a}\right\rangle \in \mathcal{H}_{a}\left|A_{a}^{\dagger}\right| \psi_{a}\right\rangle=0\right\}, \\
& \left.\mathcal{H}_{b}^{d}=\left\{\left|\psi_{b}\right\rangle \in \mathcal{H}_{b}\left|A_{b}^{\dagger}\right| \psi_{b}\right\rangle=0\right\}, \\
& \left.\mathcal{H}_{e}^{d}=\left\{\left|\psi_{e}\right\rangle \in \mathcal{H}_{e}\left|A_{b}\right| \psi_{e}\right\rangle=0\right\} .
\end{aligned}
$$

Define $\mathcal{H}_{a}^{\perp}, \mathcal{H}_{b}^{\perp}$, and $\mathcal{H}_{e}^{\perp}$ to be the complements of these spaces in $\mathcal{H}_{a}, \mathcal{H}_{b}$, and $\mathcal{H}_{e}$ :

$$
\begin{aligned}
& \mathcal{H}_{a}=\mathcal{H}_{a}^{\perp} \oplus \mathcal{H}_{a}^{d}, \\
& \mathcal{H}_{b}=\mathcal{H}_{b}^{\perp} \oplus \mathcal{H}_{b}^{d}, \\
& \mathcal{H}_{e}=\mathcal{H}_{e}^{\perp} \oplus \mathcal{H}_{e}^{d} .
\end{aligned}
$$

Clearly, states in $\mathcal{H}_{a}^{d}$ and $\mathcal{H}_{b}^{d}$ are dark states of $H$. In addition, $H$ has dark states of the form

$$
|\Lambda\rangle=\cos \phi\left|\psi_{a}\right\rangle+\sin \phi\left|\psi_{b}\right\rangle,
$$

where $\left|\psi_{a}\right\rangle \in \mathcal{H}_{a}^{\perp}$ and $\left|\psi_{b}\right\rangle \in \mathcal{H}_{b}^{\perp}$. We will call such states lambda dark states, and say that $\left|\psi_{a}\right\rangle$ and $\left|\psi_{b}\right\rangle$ form a lambda pair. Since $H|\Lambda\rangle=0$, we have that

$$
\cos \theta \sin \phi A_{b}^{\dagger}\left|\psi_{b}\right\rangle=\sin \theta \cos \phi A_{a}^{\dagger}\left|\psi_{a}\right\rangle .
$$

This equation must hold for all values of $\theta$, so $\phi$ must be related to $\theta$ by

$$
\tan \phi=\lambda \tan \theta
$$

for some value $\lambda$. Thus states $\left|\psi_{a}\right\rangle$ and $\left|\psi_{b}\right\rangle$ are related by

$$
\lambda A_{b}^{\dagger}\left|\psi_{b}\right\rangle=A_{a}^{\dagger}\left|\psi_{a}\right\rangle .
$$

Note that by using Eq. (12), we can also express $|\Lambda\rangle$ as

$$
|\Lambda\rangle=\left(\cos ^{2} \theta+\lambda^{2} \sin ^{2} \theta\right)^{-1 / 2}\left(\cos \theta\left|\psi_{a}\right\rangle+\lambda \sin \theta\left|\psi_{b}\right\rangle\right) .
$$

Define $\mathcal{H}_{a}^{\lambda}$ to be the space of states $\left|\psi_{a}\right\rangle \in \mathcal{H}_{a}^{\perp}$ such that $A_{a}^{\dagger}\left|\psi_{a}\right\rangle \in \mathcal{H}_{e}^{\perp}$, and define $\mathcal{H}_{a}^{\prime}$ to be the complement of $\mathcal{H}_{a}^{\lambda}$ in $\mathcal{H}_{a}^{\perp}$ :

$$
\mathcal{H}_{a}^{\perp}=\mathcal{H}_{a}^{\lambda} \oplus \mathcal{H}_{a}^{\prime} .
$$

Note that from the definitions of $\mathcal{H}_{a}^{\prime}$ and $\mathcal{H}_{e}^{d}$, it follows that $\operatorname{dim} \mathcal{H}_{a}^{\prime} \leq \operatorname{dim} \mathcal{H}_{e}^{d}$.

We will now show that for every normalized state $\left|\psi_{a}\right\rangle \in \mathcal{H}_{a}^{\lambda}$, we can construct a normalized state $\left|\psi_{b}\right\rangle \in \mathcal{H}_{b}^{\perp}$ such that $\left|\psi_{a}\right\rangle$ and $\left|\psi_{b}\right\rangle$ form a lambda pair. First, note that we can view the raising operator $A_{a}^{\dagger}$ as a mapping $A_{a}^{\dagger}: \mathcal{H}_{a} \rightarrow \mathcal{H}_{e}$. Since the image of $\mathcal{H}_{a}^{\lambda}$ under $A_{a}^{\dagger}$ lies entirely in $\mathcal{H}_{e}^{\perp}$, we can define a mapping $R_{a}^{\dagger}: \mathcal{H}_{a}^{\lambda} \rightarrow \mathcal{H}_{e}^{\perp}$ by

$$
R_{a}^{\dagger}\left|\psi_{a}\right\rangle \equiv A_{a}^{\dagger}\left|\psi_{a}\right\rangle
$$

where $\left|\psi_{a}\right\rangle$ is an arbitrary state in $\mathcal{H}_{a}^{\lambda}$. Similarly, given the definitions of $\mathcal{H}_{b}^{\perp}$ and $\mathcal{H}_{e}^{\perp}$, we can define mappings $R_{b}^{\dagger}: \mathcal{H}_{b}^{\perp}$ $\rightarrow \mathcal{H}_{e}^{\perp}$ and $R_{b}: \mathcal{H}_{e}^{\perp} \rightarrow \mathcal{H}_{b}^{\perp}$ by

$$
R_{b}^{\dagger}\left|\psi_{b}\right\rangle \equiv A_{b}^{\dagger}\left|\psi_{b}\right\rangle
$$

$$
R_{b}\left|\psi_{e}\right\rangle \equiv A_{b}\left|\psi_{e}\right\rangle
$$

where $\left|\psi_{b}\right\rangle$ and $\left|\psi_{e}\right\rangle$ are arbitrary states in $\mathcal{H}_{b}^{\perp}$ and $\mathcal{H}_{e}^{\perp}$. Note that the only state in $\mathcal{H}_{a}^{\lambda}$ that lies in the null space of $A_{a}^{\dagger}$ is the null state, the only state in $\mathcal{H}_{b}^{\perp}$ that lies in the null space of $A_{b}^{\dagger}$ is the null state, and the only state in $\mathcal{H}_{e}^{\perp}$ that lies in null space of $A_{b}$ is the null state; thus the null spaces of $R_{a}^{\dagger}, R_{b}^{\dagger}$, and $R_{b}$ are trivial. This means that the mapping $R_{b}^{\dagger} R_{b}: \mathcal{H}_{e}^{\perp}$ $\rightarrow \mathcal{H}_{e}^{\perp}$ is invertible, and we can define a mapping $M: \mathcal{H}_{a}^{\lambda}$ $\rightarrow \mathcal{H}_{b}^{\perp}$ by

$$
M=R_{b}\left(R_{b}^{\dagger} R_{b}\right)^{-1} R_{a}^{\dagger} .
$$

Define $\mathcal{H}_{b}^{\lambda}$ to be the image of $M$, and define $\mathcal{H}_{b}^{\prime}$ to be the complement of $\mathcal{H}_{b}^{\lambda}$ in $\mathcal{H}_{b}^{\perp}$ :

$$
\mathcal{H}_{b}^{\perp}=\mathcal{H}_{b}^{\lambda} \oplus \mathcal{H}_{b}^{\prime} .
$$

Note that because the null spaces of $R_{a}^{\dagger}, R_{b}^{\dagger}$, and $R_{b}$ are all trivial, the null space of $M$ is also trivial, and therefore $\operatorname{dim} \mathcal{H}_{b}^{\lambda}=\operatorname{dim} \mathcal{H}_{a}^{\lambda}$. Given a normalized state $\left|\psi_{a}\right\rangle \in \mathcal{H}_{a}^{\lambda}$, define $\lambda$ by

$$
\lambda=\left\langle\psi_{a}\left|M^{\dagger} M\right| \psi_{a}\right\rangle^{1 / 2},
$$

and define a normalized state $\left|\psi_{b}\right\rangle \in \mathcal{H}_{b}^{\lambda}$ by

$$
\left|\psi_{b}\right\rangle=\frac{1}{\lambda} M\left|\psi_{a}\right\rangle
$$

Using Eqs. (19) and (22), we find that

$$
\begin{aligned}
\lambda A_{b}^{\dagger}\left|\psi_{b}\right\rangle=\lambda R_{b}^{\dagger}\left|\psi_{b}\right\rangle=R_{b}^{\dagger} M\left|\psi_{a}\right\rangle & =R_{b}^{\dagger} R_{b}\left(R_{b}^{\dagger} R_{b}\right)^{-1} R_{a}^{\dagger}\left|\psi_{a}\right\rangle \\
& =R_{a}^{\dagger}\left|\psi_{a}\right\rangle=A_{a}^{\dagger}\left|\psi_{a}\right\rangle .
\end{aligned}
$$

Thus states $\left|\psi_{b}\right\rangle$ and $\left|\psi_{a}\right\rangle$ satisfy Eq. (13) and therefore form a lambda pair.

We claim that none of the states in $\mathcal{H}_{a}^{\prime}$ can form lambda pairs with states in $\mathcal{H}_{b}^{\perp}$. To see this, consider a superposition of states $\left|\psi_{a}\right\rangle \in \mathcal{H}_{a}^{\prime}$ and $\left|\psi_{b}\right\rangle \in \mathcal{H}_{b}^{\perp}$ :

$$
|\phi\rangle=c_{a}\left|\psi_{a}\right\rangle+c_{b}\left|\psi_{b}\right\rangle \text {. }
$$

Because of the way we have defined $\mathcal{H}_{a}^{\prime}$, there must be a state $\left|\psi_{e}\right\rangle \in \mathcal{H}_{e}^{d}$ such that

$$
\left\langle\psi_{e}\left|A_{a}^{\dagger}\right| \psi_{a}\right\rangle \neq 0 \text {. }
$$

Since $\left|\psi_{e}\right\rangle \in \mathcal{H}_{e}^{d}$ we have that $A_{b}\left|\psi_{e}\right\rangle=0$, so

$$
\left\langle\psi_{e}\left|A_{b}^{\dagger}\right| \psi_{b}\right\rangle=0
$$

and therefore

$$
\left\langle\psi_{e}|H| \phi\right\rangle=-\frac{\Omega}{2} \sin \theta\left\langle\psi_{e}\left|A_{a}^{\dagger}\right| \psi_{a}\right\rangle c_{a} .
$$

Thus for $c_{a} \neq 0$ and $\sin \theta \neq 0$ we have that $H|\phi\rangle \neq 0$, so $|\phi\rangle$ cannot be a lambda dark state.

Since all the states in $\mathcal{H}_{a}^{\lambda}$ form lambda pairs with states in $\mathcal{H}_{b}^{\lambda}$, and none of the states in $\mathcal{H}_{a}^{\prime}$ form lambda pairs with states in $\mathcal{H}_{b}^{\perp}$, it follows that none of the states in $\mathcal{H}_{b}^{\prime}$ form lambda pairs with states in $\mathcal{H}_{a}^{\perp}$. Thus states in $\mathcal{H}_{a}^{\prime}$ and $\mathcal{H}_{b}^{\prime}$ do not form dark states of $H$, and under adiabatic passage they are driven to the excited state manifold and scatter incoherently. 


\section{UNITARY TRANSFORMATION}

In the previous section we defined Hilbert spaces $\mathcal{H}_{a}^{\lambda}$ and $\mathcal{H}_{b}^{\lambda}$, which are subspaces of the total Hilbert spaces for ground state manifolds $a$ and $b$. We will now show that under adiabatic passage from $a$ to $b$ states in $\mathcal{H}_{a}^{\lambda}$ coherently evolve into states in $\mathcal{H}_{b}^{\lambda}$, and we will derive the unitary transformation $U: \mathcal{H}_{a}^{\lambda} \rightarrow \mathcal{H}_{b}^{\lambda}$ that describes this evolution.

First, choose an orthonormal basis $\left\{\left|\psi_{a 1}\right\rangle, \ldots,\left|\psi_{a n}\right\rangle\right\}$ for $\mathcal{H}_{a}^{\lambda}$, and use the mapping $M$ given in Eq. (19) to construct a normalized basis $\left\{\left|\psi_{b 1}\right\rangle, \ldots,\left|\psi_{b n}\right\rangle\right\}$ for $\mathcal{H}_{b}^{\lambda}$, where $\left|\psi_{b j}\right\rangle$ is defined by

$$
\begin{gathered}
\left|\psi_{b j}\right\rangle=\frac{1}{\lambda_{j}} M\left|\psi_{a j}\right\rangle, \\
\lambda_{j}=\left\langle\psi_{a j}\left|M^{\dagger} M\right| \psi_{a j}\right\rangle^{1 / 2} .
\end{gathered}
$$

Note that the basis states for $\mathcal{H}_{b}^{\lambda}$ will not necessarily be mutually orthogonal. The states $\left|\psi_{a j}\right\rangle$ and $\left|\psi_{b j}\right\rangle$ form a lambda pair and define a lambda dark state that is given by

$$
\left|\Lambda_{j}(\theta)\right\rangle=\cos \phi_{j}(\theta)\left|\psi_{a j}\right\rangle+\sin \phi_{j}(\theta)\left|\psi_{b j}\right\rangle,
$$

where

$$
\phi_{j}(\theta)=\tan ^{-1}\left(\lambda_{j} \tan \theta\right) .
$$

The states $\left\{\left|\Lambda_{1}\right\rangle, \ldots,\left|\Lambda_{n}\right\rangle\right\}$ form a basis for the lambda dark states, so we can express a general lambda dark state $|\Lambda\rangle$ as

$$
|\Lambda\rangle=\sum_{j} c_{j}\left|\Lambda_{j}\right\rangle .
$$

In the adiabatic limit, the time evolution of $|\Lambda\rangle$ is given by Schrödinger's equation:

$$
i \frac{d}{d t}|\Lambda\rangle=H|\Lambda\rangle=0 .
$$

Thus, substituting for $|\Lambda\rangle$, we find that

$$
\sum_{j}\left(\dot{c}_{j}\left|\Lambda_{j}\right\rangle+c_{j} \dot{\phi}_{j}\left|\bar{\Lambda}_{j}\right\rangle\right)=0,
$$

where the dots indicate derivatives with respect to $\theta$, and where we have defined

$$
\left|\bar{\Lambda}_{j}\right\rangle=\frac{d}{d \phi_{j}}\left|\Lambda_{j}\right\rangle=-\sin \phi_{j}\left|\psi_{a j}\right\rangle+\cos \phi_{j}\left|\psi_{b j}\right\rangle .
$$

Taking the inner product of Eq. (34) with $\left\langle\Lambda_{k}\right|$, we obtain

$$
\sum_{j}\left(\dot{c}_{j}\left\langle\Lambda_{k} \mid \Lambda_{j}\right\rangle+c_{j} \dot{\phi}_{j}\left\langle\Lambda_{k} \mid \bar{\Lambda}_{j}\right\rangle\right)=0 .
$$

Since $\left\{\left|\Lambda_{1}\right\rangle, \ldots,\left|\Lambda_{n}\right\rangle\right\}$ is a complete basis for the lambda dark states, the matrix $\left\langle\Lambda_{k} \mid \Lambda_{j}\right\rangle$ is invertible; we will denote its inverse by $L_{i k}$ :

$$
\sum_{k} L_{i k}\left\langle\Lambda_{k} \mid \Lambda_{j}\right\rangle=\delta_{i j}
$$

If we multiply Eq. (36) by $L_{i k}$ and then sum over $k$, we obtain the following equations of motion for the expansion coefficients $c_{i}$ :

$$
\dot{c}_{i}=-\sum_{j} \sum_{k} L_{i k}\left\langle\Lambda_{k} \mid \bar{\Lambda}_{j}\right\rangle \dot{\phi}_{j} c_{j} .
$$

Note that we can express an arbitrary state $\left|\psi_{a}\right\rangle \in \mathcal{H}_{a}^{\lambda}$ as

$$
\left|\psi_{a}\right\rangle=\sum_{j} c_{j}(0)\left|\psi_{a j}\right\rangle=\sum_{j} c_{j}(0)\left|\Lambda_{j}(0)\right\rangle
$$

for some set of amplitudes $c_{j}(0)$. We can then integrate Eq. (38) subject to these initial conditions to obtain a state

$$
U\left|\psi_{a}\right\rangle=\sum_{j} c_{j}(\pi / 2)\left|\Lambda_{j}(\pi / 2)\right\rangle=\sum_{j} c_{j}(\pi / 2)\left|\psi_{b j}\right\rangle .
$$

This defines the unitary transformation $U$.

\section{A. Example: $\operatorname{dim} \mathcal{H}_{a}^{\lambda}=\operatorname{dim} \mathcal{H}_{b}^{\lambda}=\mathbf{2}$}

As an example, we will write down the equations of motion explicitly for the case $\operatorname{dim} \mathcal{H}_{a}^{\lambda}=\operatorname{dim} \mathcal{H}_{b}^{\lambda}=2$. We choose an orthonormal basis of states $\left\{\left|\psi_{a 1}\right\rangle,\left|\psi_{a 2}\right\rangle\right\}$ for $\mathcal{H}_{a}^{\lambda}$, use Eq. (28) to obtain a basis of states $\left\{\left|\psi_{b 1}\right\rangle,\left|\psi_{b 2}\right\rangle\right\}$ for $\mathcal{H}_{b}^{\lambda}$, and use Eq. (29) to obtain the values $\lambda_{1}$ and $\lambda_{2}$. The matrices $\left\langle\Lambda_{k} \mid \Lambda_{j}\right\rangle$ and $\left\langle\Lambda_{k} \mid \bar{\Lambda}_{j}\right\rangle$ are given by

$$
\begin{aligned}
\left\langle\Lambda_{k} \mid \Lambda_{j}\right\rangle & =\left(\begin{array}{cc}
1 & z \sin \phi_{1} \sin \phi_{2} \\
z^{*} \sin \phi_{1} \sin \phi_{2} & 1
\end{array}\right), \\
\left\langle\Lambda_{k} \mid \bar{\Lambda}_{j}\right\rangle & =\left(\begin{array}{cc}
0 & z \sin \phi_{1} \cos \phi_{2} \\
z^{*} \cos \phi_{1} \sin \phi_{2} & 0
\end{array}\right),
\end{aligned}
$$

where

$$
z=\left\langle\psi_{b 1} \mid \psi_{b 2}\right\rangle .
$$

The inverse of $\left\langle\Lambda_{k} \mid \Lambda_{j}\right\rangle$ is

$$
\begin{aligned}
L_{i k}= & \left(1-|z|^{2} \sin ^{2} \phi_{1} \sin ^{2} \phi_{2}\right)^{-1} \\
& \times\left(\begin{array}{cc}
1 & -z \sin \phi_{1} \sin \phi_{2} \\
-z^{*} \sin \phi_{1} \sin \phi_{2} & 1
\end{array}\right) .
\end{aligned}
$$

Substituting these matrices into Eq. (38), we find that the equations of motion for $c_{1}$ and $c_{2}$ are

$$
\left(\begin{array}{c}
\dot{c}_{1} \\
\dot{c}_{2}
\end{array}\right)=\left(1-|z|^{2} \sin ^{2} \phi_{1} \sin ^{2} \phi_{2}\right)^{-1}\left(\begin{array}{cc}
|z|^{2} \sin \phi_{1} \cos \phi_{1} \sin ^{2} \phi_{2} & -z \sin \phi_{1} \cos \phi_{2} \\
-z^{*} \cos \phi_{1} \sin \phi_{2} & |z|^{2} \sin \phi_{2} \cos \phi_{2} \sin ^{2} \phi_{1}
\end{array}\right)\left(\begin{array}{c}
\dot{\phi}_{1} c_{1} \\
\dot{\phi}_{2} c_{2}
\end{array}\right),
$$


and from Eq. (31) we have that

$$
\begin{gathered}
\cos \phi_{k}=\left(\cos ^{2} \theta+\lambda_{k}^{2} \sin ^{2} \theta\right)^{-1 / 2} \cos \theta, \\
\sin \phi_{k}=\left(\cos ^{2} \theta+\lambda_{k}^{2} \sin ^{2} \theta\right)^{-1 / 2} \lambda_{k} \sin \theta, \\
\dot{\phi}_{k}=\lambda_{k}\left(\cos ^{2} \theta+\lambda_{k}^{2} \sin ^{2} \theta\right)^{-1} .
\end{gathered}
$$

Given $\lambda_{1}, \lambda_{2}$, and $z$, these equations of motion can be integrated to obtain the unitary transformation $U: \mathcal{H}_{a}^{\lambda} \rightarrow \mathcal{H}_{b}^{\lambda}$. For certain special cases, we can perform the integration analytically and write down the explicit form of $U$.

First, suppose $\left|\psi_{b 1}\right\rangle$ and $\left|\psi_{b 2}\right\rangle$ are orthogonal. Then $z=0$ and the equations of motion reduce to $\dot{c}_{1}=\dot{c}_{2}=0$, so the unitary transformation $U: \mathcal{H}_{a}^{\lambda} \rightarrow \mathcal{H}_{b}^{\lambda}$ is given by

$$
\begin{aligned}
& U\left|\psi_{a 1}\right\rangle=\left|\psi_{b 2}\right\rangle, \\
& U\left|\psi_{a 2}\right\rangle=\left|\psi_{b 2}\right\rangle .
\end{aligned}
$$

Next, suppose $\lambda_{1}=\lambda_{2}$, so there is a single angle $\phi=\phi_{1}=\phi_{2}$ that characterizes both lambda dark states. Also, for simplicity, assume that $z$ is real. Then we can express the equations of motion as

$$
\begin{aligned}
\left(\begin{array}{l}
c_{1}^{\prime} \\
c_{2}^{\prime}
\end{array}\right)= & z \sin \phi \cos \phi\left(1-z^{2} \sin ^{4} \phi\right)^{-1}\left(\begin{array}{cc}
z \sin ^{2} \phi & -1 \\
-1 & z \sin ^{2} \phi
\end{array}\right) \\
& \times\left(\begin{array}{l}
c_{1} \\
c_{2}
\end{array}\right),
\end{aligned}
$$

where primes denote derivatives with respect to $\phi$. We can decouple these equations by defining new variables $\eta_{ \pm}$ $=c_{1} \pm c_{2}$ :

$$
\eta_{ \pm}^{\prime}=\mp z \sin \phi \cos \phi\left(1 \pm z \sin ^{2} \phi\right)^{-1} \eta_{ \pm} .
$$

These equations can be integrated to give

$$
\eta_{ \pm}(\phi)=\left(1 \pm z \sin ^{2} \phi\right)^{-1 / 2} \eta_{ \pm}(0) \text {. }
$$

Thus

$$
\begin{aligned}
c_{1}(\phi)= & \frac{1}{2}\left\{\left(1+z \sin ^{2} \phi\right)^{-1 / 2}\left[c_{1}(0)+c_{2}(0)\right]\right. \\
& \left.+\left(1-z \sin ^{2} \phi\right)^{-1 / 2}\left[c_{1}(0)-c_{2}(0)\right]\right\}, \\
c_{2}(\phi)= & \frac{1}{2}\left\{\left(1+z \sin ^{2} \phi\right)^{-1 / 2}\left[c_{1}(0)+c_{2}(0)\right]\right. \\
& \left.-\left(1-z \sin ^{2} \phi\right)^{-1 / 2}\left[c_{1}(0)-c_{2}(0)\right]\right\} .
\end{aligned}
$$

For adiabatic passage from $a$ to $b$ we sweep $\theta$ from 0 to $\pi / 2$, and from Eq. (31) we see that $\phi$ also sweeps from 0 to $\pi / 2$. Thus after the adiabatic passage has been completed $\phi$ $=\pi / 2$, and the unitary transformation $U: \mathcal{H}_{a}^{\lambda} \rightarrow \mathcal{H}_{b}^{\lambda}$ is given by

$$
\begin{aligned}
& U\left|\psi_{a 1}\right\rangle=\alpha\left|\psi_{b 1}\right\rangle+\beta\left|\psi_{b 2}\right\rangle, \\
& U\left|\psi_{a 2}\right\rangle=\beta\left|\psi_{b 1}\right\rangle+\alpha\left|\psi_{b 2}\right\rangle,
\end{aligned}
$$

where

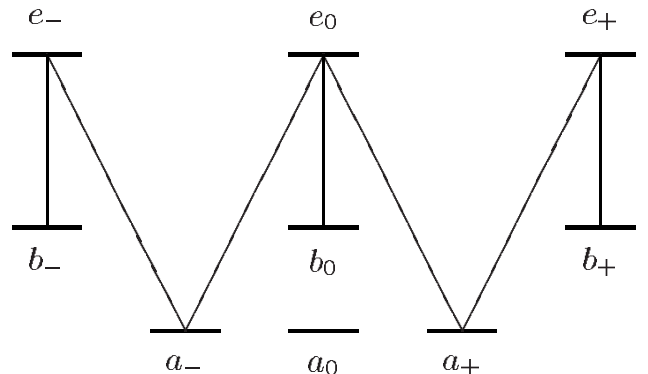

FIG. 1. Level diagram for the example system. Horizontal lines indicate states in ground state manifolds $a, b$ and excited state manifold $e$; diagonal lines indicate transitions coupled by $A_{a}$ and $A_{a}^{\dagger}$; and vertical lines indicate transitions coupled by $A_{b}$ and $A_{b}^{\dagger}$.

$$
\begin{aligned}
& \alpha=\frac{1}{2}\left[(1+z)^{-1 / 2}+(1-z)^{-1 / 2}\right], \\
& \beta=\frac{1}{2}\left[(1+z)^{-1 / 2}-(1-z)^{-1 / 2}\right] .
\end{aligned}
$$

\section{EXAMPLE SYSTEM}

We will now illustrate the formalism developed in Secs. II and III with a simple example. Consider an atom that has the following internal states:

$$
\begin{aligned}
& \mathcal{H}_{a}=\operatorname{span}\left\{\left|a_{+}\right\rangle,\left|a_{0}\right\rangle,\left|a_{-}\right\rangle\right\}, \\
& \mathcal{H}_{b}=\operatorname{span}\left\{\left|b_{+}\right\rangle,\left|b_{0}\right\rangle,\left|b_{-}\right\rangle\right\}, \\
& \mathcal{H}_{e}=\operatorname{span}\left\{\left|e_{+}\right\rangle,\left|e_{0}\right\rangle,\left|e_{-}\right\rangle\right\} .
\end{aligned}
$$

Define atomic lowering operators $A_{a}$ and $A_{b}$ by

$$
\begin{gathered}
A_{b}=\left|b_{+}\right\rangle\left\langle e_{+}|+| b_{0}\right\rangle\left\langle e_{0}|+| b_{-}\right\rangle\left\langle e_{-}\right|, \\
A_{a}=\left|a_{+}\right\rangle\left(\left\langle e_{+}\left|+\left\langle e_{0}\right|\right)+\mid a_{-}\right\rangle\left(\left\langle e_{-}\right|+\left\langle e_{0}\right|\right) .\right.
\end{gathered}
$$

The transitions coupled by these operators are shown in Fig. 1.

We will first apply the results of Sec. II to find the decompositions of Hilbert spaces $\mathcal{H}_{a}$ and $\mathcal{H}_{b}$. There is a single dark state $\left|a_{0}\right\rangle$ for the $a \rightarrow e$ transition, and there are no dark states for the $b \rightarrow e$ and $e \rightarrow b$ transitions, so

$$
\begin{gathered}
\mathcal{H}_{a}^{d}=\operatorname{span}\left\{\left|a_{0}\right\rangle\right\}, \\
\mathcal{H}_{a}^{\perp}=\operatorname{span}\left\{\left|a_{ \pm}\right\rangle\right\}, \\
\mathcal{H}_{b}^{d}=\{\}, \\
\mathcal{H}_{b}^{\perp}=\mathcal{H}_{b}, \\
\mathcal{H}_{e}^{d}=\{\}, \\
\mathcal{H}_{e}^{\perp}=\mathcal{H}_{e} .
\end{gathered}
$$

Since $A_{a}^{\dagger}\left|\psi_{a}\right\rangle \in \mathcal{H}_{e}^{\perp}$ for every state $\left|\psi_{a}\right\rangle \in \mathcal{H}_{a}^{\perp}$, we find that 


$$
\begin{gathered}
\mathcal{H}_{a}^{\lambda}=\mathcal{H}_{a}^{\perp}=\operatorname{span}\left\{\left|a_{ \pm}\right\rangle\right\}, \\
\mathcal{H}_{a}^{\prime}=\{\} .
\end{gathered}
$$

The mapping $M: \mathcal{H}_{a}^{\lambda} \rightarrow \mathcal{H}_{b}^{\perp}$ is given by

$$
M=\left(\left|b_{+}\right\rangle+\left|b_{0}\right\rangle\right)\left\langlea _ { + } \left|+\left(\left|b_{-}\right\rangle+\left|b_{0}\right\rangle\right)\left\langle a_{-}\right| .\right.\right.
$$

We can use this mapping to define states $\left|B_{ \pm}\right\rangle$that form lambda pairs with states $\left|a_{ \pm}\right\rangle$:

$$
\left|B_{ \pm}\right\rangle=\frac{1}{\lambda_{ \pm}} M\left|a_{ \pm}\right\rangle=(1 / \sqrt{2})\left(\left|b_{0}\right\rangle+\left|b_{ \pm}\right\rangle\right),
$$

where

$$
\lambda_{ \pm}=\left\langle a_{ \pm}\left|M^{\dagger} M\right| a_{ \pm}\right\rangle^{1 / 2}=\sqrt{2} .
$$

Note that $\left|B_{+}\right\rangle$and $\left|B_{-}\right\rangle$are not orthogonal:

$$
z=\left\langle B_{+} \mid B_{-}\right\rangle=1 / 2 \text {. }
$$

From the results of Sec. II, it follows that

$$
\left|\Lambda_{ \pm}\right\rangle=\cos \phi(\theta)\left|a_{ \pm}\right\rangle+\sin \phi(\theta)\left|B_{ \pm}\right\rangle
$$

are lambda dark states of $H$, where

$$
\phi(\theta)=\tan ^{-1}(\sqrt{2} \tan \theta) .
$$

Define a state $|s\rangle$ in $\mathcal{H}_{b}$ that is orthogonal to both $\left|B_{+}\right\rangle$and $\left|B_{-}\right\rangle$:

$$
|s\rangle=(1 / \sqrt{3})\left(\left|b_{-}\right\rangle-\left|b_{0}\right\rangle+\left|b_{+}\right\rangle\right) .
$$

Since the image of $M$ is $\operatorname{span}\left\{\left|B_{ \pm}\right\rangle\right\}$, we find that

$$
\begin{gathered}
\mathcal{H}_{b}^{\lambda}=\operatorname{span}\left\{\left|B_{ \pm}\right\rangle\right\}, \\
\mathcal{H}_{b}^{\prime}=\operatorname{span}\{|s\rangle\} .
\end{gathered}
$$

Now that we have decomposed the Hilbert spaces $\mathcal{H}_{a}$ and $\mathcal{H}_{b}$, let us apply the results of Sec. III to calculate the unitary transformation $U: \mathcal{H}_{a}^{\lambda} \rightarrow \mathcal{H}_{b}^{\lambda}$. We first note that a general lambda dark state of $H$ is a superposition of $\left|\Lambda_{+}\right\rangle$and $\left|\Lambda_{-}\right\rangle$:

$$
|\Lambda\rangle=c_{1}\left|\Lambda_{+}\right\rangle+c_{2}\left|\Lambda_{-}\right\rangle .
$$

Since $\lambda_{+}=\lambda_{-}$, the equations of motion for $c_{1}$ and $c_{2}$ are given by Eq. (51). As was shown in Sec. III A, these equations of motion can be integrated to give $U: \mathcal{H}_{a}^{\lambda} \rightarrow \mathcal{H}_{b}^{\lambda}$ :

$$
\begin{aligned}
& U\left|a_{+}\right\rangle=\alpha\left|B_{+}\right\rangle+\beta\left|B_{-}\right\rangle, \\
& U\left|a_{-}\right\rangle=\beta\left|B_{+}\right\rangle+\alpha\left|B_{-}\right\rangle,
\end{aligned}
$$

where $\alpha$ and $\beta$ are given by Eqs. (58) and (59) with $z=1 / 2$.

The Hilbert space decompositions and unitary transformation $U$ give a complete description of the behavior of the example system under adiabatic passage: for adiabatic passage from $a$ to $b$, states $\left|a_{ \pm}\right\rangle$coherently evolve into states $U\left|a_{ \pm}\right\rangle$and state $\left|a_{0}\right\rangle$ remains unchanged; for adiabatic passage from $b$ to $a$, states $U\left|a_{ \pm}\right\rangle$coherently evolve into states $\left|a_{ \pm}\right\rangle$, and state $|s\rangle$ is driven to the excited state manifold and scatters incoherently.

It is interesting to note that if we start in state $\left|a_{+}\right\rangle$and perform adiabatic passage from $a$ to $b$, population is trans-
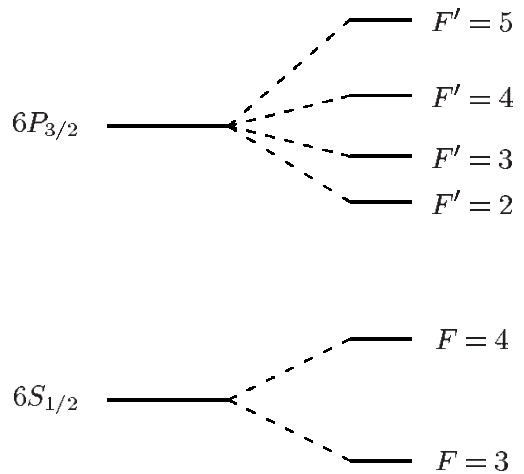

FIG. 2. Level diagram for cesium. The two hyperfine ground state manifolds $6 S_{1 / 2}, F=3$ and $6 S_{1 / 2}, F=4$ correspond to manifolds $a$ and $b$ in our theory, and one of the excited state hyperfine manifolds, either $6 P_{3 / 2}, F^{\prime}=3$ or $6 P_{3 / 2}, F^{\prime}=4$, corresponds to manifold $e$.

ferred to $\left|b_{-}\right\rangle$despite the fact that $\left\langle b_{-}\left|A_{b} A_{a}^{\dagger}\right| a_{+}\right\rangle=0$. The population reaches state $\left|b_{-}\right\rangle$by passing through state $\left|a_{-}\right\rangle$; at time $t$ the population in $\left|a_{-}\right\rangle$is given by

$$
P\left(\left|a_{-}\right\rangle\right)=\left|c_{2}(\phi)\right|^{2} \cos ^{2} \phi,
$$

where $c_{2}(\phi)$ is given by Eq. (54) with $c_{1}(0)=1, c_{2}(0)=0$, and where $\phi=\tan ^{-1}[\sqrt{2} \tan \theta(t)]$.

\section{ADIABATIC PASSAGE IN CESIUM}

In order to show how the formalism developed in Secs. II and III applies in a more physical context, we will use it to analyze adiabatic passage in the cesium atom. A level diagram for cesium is shown in Fig. 2; the two hyperfine ground state manifolds $6 S_{1 / 2}, F=3$ and $6 S_{1 / 2}, F=4$ correspond to manifolds $a$ and $b$, and one of the excited state hyperfine manifolds, either $6 P_{3 / 2}, F^{\prime}=3$ or $6 P_{3 / 2}, F^{\prime}=4$, corresponds to manifold $e$. For simplicity, we will denote the $6 S_{1 / 2}, F=3$ and $6 S_{1 / 2}, F=4$ ground state manifolds by $g_{3}$ and $g_{4}$, and the $6 P_{3 / 2}, F^{\prime}=3$ and $6 P_{3 / 2}, F^{\prime}=4$ excited state manifolds by $e_{3}$ and $e_{4}$.

Adiabatic passage between manifolds $g_{3}$ and $g_{4}$ is performed by driving the atom with a pair of classical fields that connect these manifolds to the excited state manifold $e_{F^{\prime}}$. The field driving the $g_{F} \leftrightarrow e_{F^{\prime}}$ transition corresponds to an atomic lowering operator that is given by

$$
A_{F}=\vec{A}_{F} \cdot \hat{\boldsymbol{\epsilon}}_{F},
$$

where $\hat{\epsilon}_{F}$ is the polarization of the field. The operator $\vec{A}_{F}$ is defined by

$$
\vec{A}_{F}^{\dagger} \equiv \sum_{m^{\prime}} \sum_{m} \sum_{q=-1}^{1} \beta\left(F^{\prime}, F\right)\left\langle F^{\prime}, m^{\prime} \mid 1, q ; F, m\right\rangle\left|F^{\prime}, m^{\prime}\right\rangle\langle F, m| \hat{e}_{q}^{*},
$$

where $\left\langle F^{\prime}, m^{\prime} \mid 1, q ; F, m\right\rangle$ is the Clebsch-Gordan coefficient that connects ground state $|F, m\rangle$ to excited state $\left|F^{\prime}, m^{\prime}\right\rangle$ via polarization $\hat{e}_{q}^{*}$,

$$
\hat{e}_{ \pm 1}=\mp \frac{1}{\sqrt{2}}(\hat{x} \pm i \hat{y})
$$




$$
\hat{e}_{0}=\hat{z}
$$

is a orthonormal basis of polarization vectors, and $\beta\left(F^{\prime}, F\right)$ is given by the following:

$$
\begin{array}{ccccc}
F^{\prime} & 3 & 3 & 4 & 4 \\
F & 3 & 4 & 3 & 4 \\
\beta\left(F^{\prime}, F\right) & \sqrt{3 / 4} & \sqrt{1 / 4} & \sqrt{5 / 12} & \sqrt{7 / 12}
\end{array} .
$$

In the following sections we apply the results of Sec. II to perform the Hilbert space decompositions given in Eqs. (2) and (3) for adiabatic passage via both the $F^{\prime}=3$ and $F^{\prime}=4$ excited state manifolds.

\section{A. Adiabatic passage via the $F^{\prime}=3$ manifold}

For the $g_{4} \leftrightarrow e_{3}$ transition there are two dark states $\left|d_{4 e}\right\rangle$, $\left|d_{4 o}\right\rangle$ in $g_{4}$ (these dark states are calculated in the Appendix), and there are no dark states in $e_{3}$, so

$$
\begin{gathered}
\mathcal{H}_{4}^{d}=\operatorname{span}\left\{\left|d_{4 e}\right\rangle,\left|d_{4 o}\right\rangle\right\}, \\
\mathcal{H}_{e}^{d}=\{\} .
\end{gathered}
$$

For the $g_{3} \leftrightarrow e_{3}$ transition there is a single dark state $\left|d_{3}\right\rangle$ in $g_{3}$ (this dark state is calculated in the Appendix), so

$$
\mathcal{H}_{3}^{d}=\operatorname{span}\left\{\left|d_{3}\right\rangle\right\}
$$

From the dimensions of $\mathcal{H}_{3}^{d}, \mathcal{H}_{4}^{d}$, and $\mathcal{H}_{e}^{d}$, we can work out the dimensions of all the Hilbert spaces in the decompositions of $\mathcal{H}_{3}$ and $\mathcal{H}_{4}$. First, note that because $\operatorname{dim} \mathcal{H}_{e}^{d}=0$ and $\operatorname{dim} \mathcal{H}_{3}^{\prime} \leq \operatorname{dim} \mathcal{H}_{e}^{d}$, we must have that $\operatorname{dim} \mathcal{H}_{3}^{\prime}=0$. Next, since the dimensions of $\mathcal{H}_{3}^{\lambda}, \mathcal{H}_{3}^{d}$, and $\mathcal{H}_{3}^{\prime}$ must sum to 7 , we find that $\operatorname{dim} \mathcal{H}_{3}^{\lambda}=6$. Finally, since $\operatorname{dim} \mathcal{H}_{4}^{\lambda}=\operatorname{dim} \mathcal{H}_{3}^{\lambda}=6$ and the dimensions of $\mathcal{H}_{4}^{\lambda}, \mathcal{H}_{4}^{d}$, and $\mathcal{H}_{4}^{\prime}$ must sum to 9 , we find that $\operatorname{dim} \mathcal{H}_{4}^{\prime}=1$. Thus the dimensions of the Hilbert spaces are

$$
\begin{array}{cccc}
F & \operatorname{dim} \mathcal{H}_{F}^{\lambda} & \operatorname{dim} \mathcal{H}_{F}^{d} & \operatorname{dim} \mathcal{H}_{F}^{\prime} \\
3 & 6 & 1 & 0 \\
4 & 6 & 2 & 1
\end{array} .
$$

Note that these dimensions are independent of the polarizations of the classical fields.

\section{Example: $\hat{\boldsymbol{\epsilon}}_{3}=\hat{\boldsymbol{\epsilon}}_{4}=\hat{z}$}

The Hilbert space decompositions are

$$
\begin{gathered}
\mathcal{H}_{3}^{d}=\operatorname{span}\{|3,0\rangle\}, \\
\mathcal{H}_{3}^{\prime}=\{\}, \\
\mathcal{H}_{3}^{\lambda}=\operatorname{span}\{|3, \pm 1\rangle,|3, \pm 2\rangle,|3, \pm 3\rangle\}, \\
\mathcal{H}_{4}^{d}=\operatorname{span}\{|4, \pm 4\rangle\}, \\
\mathcal{H}_{4}^{\prime}=\operatorname{span}\{|4,0\rangle\}, \\
\mathcal{H}_{4}^{\lambda}=\operatorname{span}\{|4, \pm 1\rangle,|4, \pm 2\rangle,|4, \pm 3\rangle\} .
\end{gathered}
$$

States $|3, m\rangle$ and $|4, m\rangle$ are lambda pairs for $m$ $= \pm 1, \pm 2, \pm 3$. Since the lambda dark states corresponding to these pairs are mutually orthogonal, Eq. (38) decouples and the unitary transformation $U: \mathcal{H}_{3}^{\lambda} \rightarrow \mathcal{H}_{4}^{\lambda}$ can be written down explicitly:

$$
U=\sum_{m}|4, m\rangle\langle 3, m|,
$$

where the sum is taken over $m= \pm 1, \pm 2, \pm 3$.

$$
\text { 2. Example: } \hat{\boldsymbol{\epsilon}}_{3}=\hat{z}, \hat{\boldsymbol{\epsilon}}_{4}=\hat{x}
$$

The Hilbert space decompositions are

$$
\mathcal{H}_{3}^{d}=\operatorname{span}\{|3,0\rangle\},
$$

$$
\mathcal{H}_{3}^{\prime}=\{\}
$$

$$
\begin{gathered}
\mathcal{H}_{3}^{\lambda}=\operatorname{span}\{|3, \pm 1\rangle,|3, \pm 2\rangle,|3, \pm 3\rangle\} \\
\mathcal{H}_{4}^{d}=\operatorname{span}\left\{\left|d_{4 e}\right\rangle,\left|d_{4 o}\right\rangle\right\} \\
\mathcal{H}_{4}^{\prime}=\operatorname{span}\{|s\rangle\} \\
\mathcal{H}_{4}^{\lambda}=\operatorname{span}\left\{\left|B_{ \pm 1}\right\rangle,\left|B_{ \pm 2}\right\rangle,\left|B_{ \pm 3}\right\rangle\right\}
\end{gathered}
$$

where

$$
|s\rangle \equiv(1 / \sqrt{2})(|4,1\rangle-|4,-1\rangle)
$$

and the dark states are given by

$$
\begin{aligned}
\left|d_{4 e}\right\rangle= & (1 / 8 \sqrt{2})(|4,4\rangle+\sqrt{28}|4,2\rangle+\sqrt{70}|4,0\rangle+\sqrt{28}|4,-2\rangle \\
& +|4,-4\rangle), \\
\left|d_{4 o}\right\rangle & =(1 / 4)(|4,3\rangle+\sqrt{7}|4,1\rangle+\sqrt{7}|4,-1\rangle+|4,-3\rangle) .
\end{aligned}
$$

We have defined states $\left|B_{ \pm 1}\right\rangle,\left|B_{ \pm 2}\right\rangle$, and $\left|B_{ \pm 3}\right\rangle$ that form lambda pairs with states $|3, \pm 1\rangle,|3, \pm 2\rangle$, and $|3, \pm 3\rangle$; they are given by

$$
\begin{aligned}
\left|B_{ \pm 3}\right\rangle= & (1 / 8 \sqrt{254})(127|4, \pm 4\rangle-2 \sqrt{7}|4, \pm 2\rangle-\sqrt{70}|4,0\rangle \\
- & 2 \sqrt{7}|4, \mp 2\rangle-|4, \mp 4\rangle) \\
\left|B_{ \pm 2}\right\rangle= & (1 / 4 \sqrt{15})(15|4, \pm 3\rangle-\sqrt{7}|4, \pm 1\rangle-\sqrt{7}|4, \mp 1\rangle \\
- & |4, \mp 3\rangle) \\
\left|B_{ \pm 1}\right\rangle= & (1 / 24 \sqrt{638})(99|4, \pm 4\rangle+198 \sqrt{7}|4, \pm 2\rangle \\
& -29 \sqrt{70}|4,0\rangle-58 \sqrt{7}|4, \mp 2\rangle-29|4, \mp 4\rangle)
\end{aligned}
$$

and the corresponding $\lambda$ values are

$$
\begin{gathered}
\lambda_{ \pm 3}=\sqrt{10287 / 1792}, \\
\lambda_{ \pm 2}=\sqrt{45 / 14}, \\
\lambda_{ \pm 1}=\sqrt{8613 / 8960} .
\end{gathered}
$$

The unitary transformation $U: \mathcal{H}_{3}^{\lambda} \rightarrow \mathcal{H}_{4}^{\lambda}$ can be obtained by numerically integrating Eq. (38) using the above expressions 
for the states and $\lambda$ values. We can say something about the structure of this unitary transformation by noting that the polarizations of the classical fields impose the selection rule $\Delta m= \pm 1$. Thus it is useful to decompose $\mathcal{H}_{3}^{\lambda}$ and $\mathcal{H}_{4}^{\lambda}$ into subspaces of even and odd Zeeman states:

$$
\begin{aligned}
& \mathcal{H}_{3}^{\lambda}=\mathcal{H}_{3}^{\lambda e} \oplus \mathcal{H}_{3}^{\lambda o}, \\
& \mathcal{H}_{4}^{\lambda}=\mathcal{H}_{4}^{\lambda e} \oplus \mathcal{H}_{4}^{\lambda o},
\end{aligned}
$$

where

$$
\begin{gathered}
\mathcal{H}_{3}^{\lambda e}=\operatorname{span}\{|3, \pm 2\rangle\}, \\
\mathcal{H}_{3}^{\lambda o}=\operatorname{span}\{|3, \pm 1\rangle,|3, \pm 3\rangle\}, \\
\mathcal{H}_{4}^{\lambda o}=\operatorname{span}\left\{\left|B_{ \pm 2}\right\rangle\right\}, \\
\mathcal{H}_{4}^{\lambda e}=\operatorname{span}\left\{\left|B_{ \pm 1}\right\rangle,\left|B_{ \pm 3}\right\rangle\right\} .
\end{gathered}
$$

The selection rule implies that $U$ maps $\mathcal{H}_{3}^{\lambda e}$ to $\mathcal{H}_{4}^{\lambda o}$ and $\mathcal{H}_{3}^{\lambda o}$ to $\mathcal{H}_{4}^{\lambda e}$. Since $\operatorname{dim} \mathcal{H}_{3}^{\lambda e}=\operatorname{dim} \mathcal{H}_{4}^{\lambda o}=2$ and $\lambda_{+2}=\lambda_{-2}$, we can use the results of Sec. III A to write down the unitary transformation $U: \mathcal{H}_{3}^{\lambda e} \rightarrow \mathcal{H}_{4}^{\lambda o}$ explicitly:

$$
\begin{gathered}
U|3,+2\rangle=\alpha\left|B_{+2}\right\rangle+\beta\left|B_{-2}\right\rangle, \\
U|3,-2\rangle=\beta\left|B_{+2}\right\rangle+\alpha\left|B_{-2}\right\rangle,
\end{gathered}
$$

where $\alpha$ and $\beta$ are given by Eqs. (58) and (59) with $z$ $=\left\langle B_{+2} \mid B_{-2}\right\rangle=-1 / 15$.

\section{B. Adiabatic passage via the $F^{\prime}=4$ manifold}

For the $g_{4} \leftrightarrow e_{4}$ transition there is one dark state $\left|d_{4}\right\rangle$ in $g_{4}$ and one dark state $\left|d_{e}\right\rangle$ in $e_{4}$ (these dark states are calculated in the Appendix), so

$$
\begin{aligned}
& \mathcal{H}_{4}^{d}=\operatorname{span}\left\{\left|d_{4}\right\rangle\right\}, \\
& \mathcal{H}_{e}^{d}=\operatorname{span}\left\{\left|d_{e}\right\rangle\right\} .
\end{aligned}
$$

For the $g_{3} \leftrightarrow e_{4}$ transition there are no dark states in $g_{3}$, so

$$
\mathcal{H}_{3}^{d}=\{\} .
$$

As with adiabatic passage via $F^{\prime}=3$, we can use the dimensions of $\mathcal{H}_{3}^{d}, \mathcal{H}_{4}^{d}$, and $\mathcal{H}_{e}^{d}$ to say something about the dimensions of the other Hilbert spaces in the decomposition of $\mathcal{H}_{3}$ and $\mathcal{H}_{4}$. For adiabatic passage via $F^{\prime}=4$, however, there are two separate cases to consider: since $\operatorname{dim} \mathcal{H}_{3}^{\prime} \leq \operatorname{dim} \mathcal{H}_{e}^{d}$ and $\operatorname{dim} \mathcal{H}_{e}^{d}=1$, we find that $\operatorname{dim} \mathcal{H}_{3}^{\prime}$ can be either 0 or 1 . For $\operatorname{dim} \mathcal{H}_{3}^{\prime}=0$ the dimensions of the Hilbert spaces are

$$
\begin{array}{cccc}
F & \operatorname{dim} \mathcal{H}_{F}^{\lambda} & \operatorname{dim} \mathcal{H}_{F}^{d} & \operatorname{dim} \mathcal{H}_{F}^{\prime} \\
3 & 7 & 0 & 0 \\
4 & 7 & 1 & 1
\end{array}
$$

and for $\operatorname{dim} \mathcal{H}_{3}^{\prime}=1$ the dimensions of the Hilbert spaces are

$\begin{array}{cccc}F & \operatorname{dim} \mathcal{H}_{F}^{\lambda} & \operatorname{dim} \mathcal{H}_{F}^{d} & \operatorname{dim} \mathcal{H}_{F}^{\prime} \\ 3 & 6 & 0 & 1 \\ 4 & 6 & 1 & 2\end{array}$.

Thus for adiabatic passage via $F^{\prime}=4$ the dimensions of the Hilbert spaces depend on the polarizations of the classical fields.

\section{Example: $\hat{\boldsymbol{\epsilon}}_{3}=\hat{\boldsymbol{\epsilon}}_{4}=\hat{z}$}

For this example $\operatorname{dim} \mathcal{H}_{3}^{\prime}=1$, and the Hilbert space decompositions are

$$
\begin{gathered}
\mathcal{H}_{3}^{d}=\{\}, \\
\mathcal{H}_{3}^{\prime}=\operatorname{span}\{|3,0\rangle\}, \\
\mathcal{H}_{3}^{\lambda}=\operatorname{span}\{|3, \pm 1\rangle,|3, \pm 2\rangle,|3, \pm 3\rangle\}, \\
\mathcal{H}_{4}^{d}=\operatorname{span}\{|4,0\rangle\}, \\
\mathcal{H}_{4}^{\prime}=\operatorname{span}\{|4, \pm 4\rangle\}, \\
\mathcal{H}_{4}^{\lambda}=\operatorname{span}\{|4, \pm 1\rangle,|4, \pm 2\rangle,|4, \pm 3\rangle\} .
\end{gathered}
$$

States $|3, m\rangle$ and $|4, m\rangle$ are lambda pairs for $m$ $= \pm 1, \pm 2, \pm 3$. Since the lambda dark states corresponding to these pairs are mutually orthogonal, Eq. (38) decouples, and the unitary transformation $U: \mathcal{H}_{3}^{\lambda} \rightarrow \mathcal{H}_{4}^{\lambda}$ can be written down explicitly:

$$
U=\sum_{m}|4, m\rangle\langle 3, m|,
$$

where the sum is taken over $m= \pm 1, \pm 2, \pm 3$.

$$
\text { 2. Example: } \hat{\boldsymbol{\epsilon}}_{3}=\hat{\boldsymbol{x}}, \hat{\boldsymbol{\epsilon}}_{4}=\hat{z}
$$

For this example $\operatorname{dim} \mathcal{H}_{3}^{\prime}=1$, and the Hilbert space decompositions are

$$
\begin{gathered}
\mathcal{H}_{3}^{d}=\{\}, \\
\mathcal{H}_{3}^{\prime}=\operatorname{span}\{|-\rangle\}, \\
\mathcal{H}_{3}^{\lambda}=\operatorname{span}\{|3, \pm 2\rangle,|3, \pm 3\rangle,|3,0\rangle,|+\rangle\}, \\
\mathcal{H}_{4}^{d}=\operatorname{span}\{|4,0\rangle\}, \\
\mathcal{H}_{4}^{\prime}=\operatorname{span}\left\{\left|s_{e}\right\rangle,\left|s_{o}\right\rangle\right\}, \\
\mathcal{H}_{4}^{\lambda}=\operatorname{span}\left\{\left|B_{ \pm 2}\right\rangle,\left|B_{ \pm 3}\right\rangle,\left|B_{0}\right\rangle,\left|B_{+}\right\rangle\right\},
\end{gathered}
$$

where

$$
| \pm\rangle \equiv(1 / \sqrt{2})(|3,1\rangle \pm|3,-1\rangle)
$$

and

$$
\left|s_{e}\right\rangle \equiv(1 / 4)(|4,4\rangle+\sqrt{7}|4,2\rangle-\sqrt{7}|4,-2\rangle-|4,-4\rangle),
$$




$$
\left|s_{o}\right\rangle \equiv(1 / \sqrt{32})(3|4,3\rangle+\sqrt{7}|4,1\rangle-\sqrt{7}|4,-1\rangle-3|4,-3\rangle) .
$$

We have defined states $\left|B_{+}\right\rangle,\left|B_{0}\right\rangle,\left|B_{ \pm 2}\right\rangle$, and $\left|B_{ \pm 3}\right\rangle$ that form lambda pairs with states $|+\rangle,|3,0\rangle,|3, \pm 2\rangle$, and $|3, \pm 3\rangle$; they are given by

$$
\begin{gathered}
\left|B_{ \pm 3}\right\rangle=(1 / \sqrt{8})(\sqrt{7}|4, \pm 4\rangle-|4, \pm 2\rangle), \\
\left|B_{ \pm 2}\right\rangle=(1 / 4)(\sqrt{7}|4, \pm 3\rangle-3|4, \pm 1\rangle), \\
\left|B_{0}\right\rangle=(1 / \sqrt{2})(|4,1\rangle+|4,-1\rangle), \\
\left|B_{+}\right\rangle=(1 / \sqrt{2})(|4,2\rangle+|4,-2\rangle),
\end{gathered}
$$

and the corresponding $\lambda$ values are

$$
\begin{gathered}
\lambda_{ \pm 3}=\sqrt{25 / 49}, \\
\lambda_{ \pm 2}=\sqrt{200 / 147}, \\
\lambda_{0}=\sqrt{250 / 49}, \\
\lambda_{+}=\sqrt{375 / 392} .
\end{gathered}
$$

The unitary transformation $U: \mathcal{H}_{3}^{\lambda} \rightarrow \mathcal{H}_{4}^{\lambda}$ can be obtained by numerically integrating Eq. (38) using the above expressions for the states and $\lambda$ values. As with the example given in Sec. $\mathrm{V} \mathrm{A} 2$, the polarizations of the classical fields impose the selection rule $\Delta m= \pm 1$, so it is useful to decompose $\mathcal{H}_{3}^{\lambda}$ and $\mathcal{H}_{4}^{\lambda}$ into subspaces of even and odd Zeeman states:

$$
\begin{aligned}
& \mathcal{H}_{3}^{\lambda}=\mathcal{H}_{3}^{\lambda e} \oplus \mathcal{H}_{3}^{\lambda o}, \\
& \mathcal{H}_{4}^{\lambda}=\mathcal{H}_{4}^{\lambda e} \oplus \mathcal{H}_{4}^{\lambda o},
\end{aligned}
$$

where

$$
\begin{gathered}
\mathcal{H}_{3}^{\lambda e}=\operatorname{span}\{|3,0\rangle,|3, \pm 2\rangle\}, \\
\mathcal{H}_{3}^{\lambda o}=\operatorname{span}\{|+\rangle,|3, \pm 3\rangle\}, \\
\mathcal{H}_{4}^{\lambda o}=\operatorname{span}\left\{\left|B_{0}\right\rangle,\left|B_{ \pm 2}\right\rangle\right\}, \\
\mathcal{H}_{4}^{\lambda e}=\operatorname{span}\left\{\left|B_{+}\right\rangle,\left|B_{ \pm 3}\right\rangle\right\} .
\end{gathered}
$$

The selection rule implies that $U$ maps $\mathcal{H}_{3}^{\lambda e}$ to $\mathcal{H}_{4}^{\lambda o}$ and $\mathcal{H}_{3}^{\lambda o}$ to $\mathcal{H}_{4}^{\lambda e}$.

\section{Example: $\hat{\boldsymbol{\epsilon}}_{3}=\hat{\boldsymbol{\epsilon}}_{4}=\hat{\boldsymbol{e}}_{+1}$}

For this example $\operatorname{dim} \mathcal{H}_{3}^{\prime}=0$, and the Hilbert space decompositions are

$$
\begin{gathered}
\mathcal{H}_{3}^{d}=\{\}, \\
\mathcal{H}_{3}^{\prime}=\operatorname{span}\{\}, \\
\mathcal{H}_{3}^{\lambda}=\operatorname{span}\{|3,0\rangle,|3, \pm 1\rangle,|3, \pm 2\rangle,|3, \pm 3\rangle\},
\end{gathered}
$$

$$
\begin{gathered}
\mathcal{H}_{4}^{d}=\operatorname{span}\{|4,4\rangle\}, \\
\mathcal{H}_{4}^{\prime}=\operatorname{span}\{|4,-4\rangle\}, \\
\mathcal{H}_{4}^{\lambda}=\operatorname{span}\{|4,0\rangle,|4, \pm 1\rangle,|4, \pm 2\rangle,|4, \pm 3\rangle\} .
\end{gathered}
$$

States $|3, m\rangle$ and $|4, m\rangle$ are lambda pairs for $m$ $=0, \pm 1, \pm 2, \pm 3$. Since the lambda dark states corresponding to these pairs are mutually orthogonal, Eq. (38) decouples, and the unitary transformation $U: \mathcal{H}_{3}^{\lambda} \rightarrow \mathcal{H}_{4}^{\lambda}$ can be written down explicitly:

$$
U=\sum_{m=-3}^{3}|4, m\rangle\langle 3, m|
$$

\section{CONCLUSION}

We have presented a general formalism for describing stimulated Raman adiabatic passage in a multilevel atom with ground state manifolds $a$ and $b$ and excited state manifold $e$, where each manifold consists of an arbitrary number of degenerate states. This formalism describes how the Hilbert spaces for manifolds $a$ and $b$ decompose into subspaces, each of which evolves in its own characteristic way under adiabatic passage. In particular, we identified subspaces $\mathcal{H}_{a}^{\lambda}$ and $\mathcal{H}_{b}^{\lambda}$ that coherently evolve into one another under adiabatic passage and calculated the unitary transformation that describes this evolution. We have illustrated the formalism with a simple toy model and used it to analyze adiabatic passage in the cesium atom. The formalism gives a complete description of the adiabatic passage process and should be useful for analyzing adiabatic passage in a wide variety of atomic systems.

\section{ACKNOWLEDGMENTS}

The author would like to thank A. Boca and T. E. Northup for helpful suggestions.

\section{APPENDIX: DARK STATES}

Here we calculate the dark states for an optical field driving an $F \rightarrow F$ or $F \rightarrow F-1$ transition. We will choose a coordinate system such that the field propagates along the $\hat{z}$ axis, and define a basis of Zeeman states relative to this quantization axis. The polarization $\hat{\epsilon}$ of the field can be expressed as

$$
\hat{\epsilon}=\alpha_{+} \hat{e}_{+1}+\alpha_{-} \hat{e}_{-1},
$$

where $\hat{e}_{ \pm 1}$ are given by Eq. (88). If $|d\rangle$ is a dark state of the field, then

$$
\hat{\boldsymbol{\epsilon}} \cdot \vec{A}^{\dagger}|d\rangle=0
$$

Thus for every state $\left|F^{\prime}, m^{\prime}\right\rangle$ in the excited state manifold, we have that

$$
\sum_{m}\left\langle F^{\prime}, m^{\prime}\left|\hat{\boldsymbol{\epsilon}} \cdot \vec{A}^{\dagger}\right| F, m\right\rangle c_{m}=0,
$$

where 


$$
c_{m} \equiv\langle F, m \mid d\rangle \text {. }
$$

Using Eq. (87) to substitute for $\vec{A}_{F}^{\dagger}$, we obtain the following dark state conditions:

$$
\begin{aligned}
& \left\langle F^{\prime}, m \mid 1,+1 ; F, m-1\right\rangle \alpha_{+} c_{m-1}+\left\langle F^{\prime}, m \mid 1,-1 ; F, m+1\right\rangle \alpha_{-} c_{m+1} \\
& \quad=0
\end{aligned}
$$

for $-F^{\prime} \leq m \leq F^{\prime}$. We will now consider $F \rightarrow F$ and $F \rightarrow F$ -1 transitions as separate cases.

\section{Dark state for an $F \rightarrow F$ transition}

The Clebsch-Gordan coefficients for $-F<m<F$ are given by

$$
\begin{aligned}
& \langle F, m \mid 1,+1 ; F, m-1\rangle=-\left(\frac{(F+m)(F+1-m)}{2 F(F+1)}\right)^{1 / 2}, \\
& \langle F, m \mid 1,-1 ; F, m+1\rangle=+\left(\frac{(F-m)(F+1+m)}{2 F(F+1)}\right)^{1 / 2} .
\end{aligned}
$$

Thus the dark state conditions are

$$
\sqrt{(F+m)(F+1-m)} \alpha_{+} c_{m-1}=\sqrt{(F-m)(F+1+m)} \alpha_{-} c_{m+1}
$$

for $-F<m<F$, and

$$
\alpha_{+} c_{F-1}=\alpha_{-} c_{-(F-1)}=0 .
$$

For each value of $F$ there is single dark state that meets these conditions. The dark state for a $3 \rightarrow 3$ transition is

$$
\begin{aligned}
\left|d_{3}\right\rangle= & N\left(\sqrt{5} \alpha_{+}^{3}|3,3\rangle+\sqrt{3} \alpha_{+}^{2} \alpha_{-}|3,1\rangle+\sqrt{3} \alpha_{+} \alpha_{-}^{2}|3,-1\rangle\right. \\
& \left.+\sqrt{5} \alpha_{-}^{3}|3,-3\rangle\right),
\end{aligned}
$$

and the dark state for a $4 \rightarrow 4$ transition is

$$
\begin{aligned}
\left|d_{4}\right\rangle= & N\left(\sqrt{35} \alpha_{+}^{4}|4,4\rangle+\sqrt{20} \alpha_{+}^{3} \alpha_{-}|4,2\rangle+\sqrt{18} \alpha_{+}^{2} \alpha_{-}^{2}|4,0\rangle\right. \\
& \left.+\sqrt{20} \alpha_{+} \alpha_{-}^{3}|4,-2\rangle+\sqrt{35} \alpha_{-}^{4}|4,-4\rangle\right)
\end{aligned}
$$

where $N$ is a normalization constant that depends on $\alpha_{ \pm}$.

\section{Dark states for an $F \rightarrow F-1$ transition}

The Clebsch-Gordan coefficients for $-F<m<F$ are given by

$$
\langle F-1, m \mid 1,+1 ; F, m-1\rangle=\left(\frac{(F-m)(F+1-m)}{2 F(2 F+1)}\right)^{1 / 2},
$$

$$
\langle F-1, m \mid 1,-1 ; F, m+1\rangle=\left(\frac{(F+m)(F+1+m)}{2 F(2 F+1)}\right)^{1 / 2} .
$$

Thus the dark state conditions are

$$
\sqrt{(F-m)(F+1-m)} \alpha_{+} c_{m-1}=-\sqrt{(F+m)(F+1+m)} \alpha_{-} c_{m+1}
$$

for $-F<m<F$. For each value of $F$ there are two dark states that meet these conditions. The dark states for a $4 \rightarrow 3$ transition are

$$
\begin{aligned}
\left|d_{4 e}\right\rangle= & N\left(\alpha_{+}^{4}|4,4\rangle-\sqrt{28} \alpha_{+}^{3} \alpha_{-}|4,2\rangle+\sqrt{70} \alpha_{+}^{2} \alpha_{-}^{2}|4,0\rangle\right. \\
& \left.-\sqrt{28} \alpha_{+} \alpha_{-}^{3}|4,-2\rangle+\alpha_{-}^{4}|4,-4\rangle\right), \\
\left|d_{4 o}\right\rangle= & N\left(\alpha_{+}^{3}|4,3\rangle-\sqrt{7} \alpha_{+}^{2} \alpha_{-}|4,1\rangle+\sqrt{7} \alpha_{+} \alpha_{-}^{2}|4,-1\rangle\right. \\
& \left.-\alpha_{-}^{3}|4,-3\rangle\right),
\end{aligned}
$$

where $N$ is a normalization constant that depends on $\alpha_{ \pm}$.
[1] F. T. Hioe, Phys. Lett. 99A, 150 (1983).

[2] J. Oreg, F. T. Hioe, and J. H. Eberly, Phys. Rev. A 29, 690 (1984).

[3] J. R. Kuklinski, U. Gaubatz, F. T. Hioe, and K. Bergmann, Phys. Rev. A 40, 6741 (1989).

[4] U. Gaubatz, P. Rudecki, M. Becker, S. Schiemann, M. Külz, and K. Bergmann, Chem. Phys. Lett. 149, 463 (1988).

[5] U. Gaubatz, P. Rudecki, S. Schiemann, and K. Bergmann, J. Chem. Phys. 92, 5363 (1990).

[6] H. G. Rubahn, E. Konz, S. Schiemann, and K. Bergmann, Z. Phys. D 22, 401 (1991).

[7] B. Broers, H. B. van Linden van den Heuvell, and L. D. Noordam, Phys. Rev. Lett. 69, 2062 (1992).

[8] S. Schiemann, A. Kuhn, S. Steuerwald, and K. Bergmann, Phys. Rev. Lett. 71, 3637 (1993).

[9] M. Weitz, B. C. Young, and S. Chu, Phys. Rev. Lett. 73, 2563 (1994).
[10] A. D. Boozer, A. Boca, R. Miller, T. E. Northup, and H. J. Kimble, Phys. Rev. Lett. 98, 193601 (2007).

[11] P. Marte, P. Zoller, and J. L. Hall, Phys. Rev. A 44, R4118 (1991).

[12] R. G. Unanyan, M. Fleischhaur, B. W. Shore, and K. Bergmann, Opt. Commun. 155, 144 (1998).

[13] H. Theuer, R. Unanyan, C. Habscheid, K. Klein, and K. Bergmann, Opt. Express 4, 77 (1999).

[14] R. G. Unanyan, B. W. Shore, and K. Bergmann, Phys. Rev. A 59, 2910 (1999).

[15] B. W. Shore, J. Martin, M. P. Fewell, and K. Bergmann, Phys. Rev. A 52, 566 (1995).

[16] J. Martin, B. W. Shore, and K. Bergmann, Phys. Rev. A 52, 583 (1995).

[17] Z. Kis and S. Stenholm, Phys. Rev. A 64, 063406 (2001).

[18] Z. Kis, A. Karpati, B. W. Shore, and N. V. Vitanov, Phys. Rev. A 70, 053405 (2004). 
[19] B. Y. Chang, I. R. Solá, V. S. Malinovsky, and J. Santamaria, Phys. Rev. A 64, 033420 (2001).

[20] Z. Kis and S. Stenholm, J. Mod. Opt. 49, 111 (2002).

[21] A. Karpati and Z. Kis, J. Phys. B 36, 905 (2003).
[22] Z. Kis, N. V. Vitanov, A. Karpati, C. Barthel, and K. Bergmann, Phys. Rev. A 72, 033403 (2005).

[23] I. Thanopulos, P. Král, and M. Shapiro, Phys. Rev. Lett. 92, 113003 (2004). 